\title{
Análise da estabilidade do escoamento de jato viscoelástico bidimensional
}

\author{
Rafael L. Sterza ${ }^{1}$ \\ Analice C. Brandi ${ }^{2}$ \\ DMC/FCT-UNESP, Presidente Prudente, SP \\ Márcio T. Mendonça ${ }^{3}$ \\ CTA/IAE/APA, São José dos Campos, SP \\ Leandro F. de Souza ${ }^{4}$ \\ ICMC/USP, São Carlos, SP
}

\begin{abstract}
Resumo. Há um grande interesse no estudo do escoamento de jato pela considerável importância tecnológica, por exemplo, processos de mistura de componentes, transferência de calor e sistemas de propulsão são algumas das aplicações industriais que envolvem esse tipo de escoamento. A otimização destes processos pode ser realizada através do controle de determinados tipos de instabilidades e vórtices, que são responsáveis por influenciar fortemente a dinâmica do jato. Ao estudar a instabilidade hidrodinâmica, através da Teoria de Estabilidade Linear, é necessário conhecer os componentes do escoamento base. Diante disso, este trabalho considera algumas hipóteses impostas sobre o escoamento base de jato laminar e, para verificá-las, utilizou-se o software OpenFOAM. Além disso, investiga-se as instabilidades de Kelvin-Helmholtz através de curvas neutras de estabilidade.
\end{abstract}

Palavras-chave. Escoamento de Jato, Software OpenFOAM, Teoria de Estabilidade Linear, Instabilidade de Kelvin-Helmholtz.

\section{Introdução}

A instabilidade viscoelástica em vários regimes de escoamento, como escoamento de cisalhamento paralelo, escoamento entre placas e escoamento de Taylor-Couette, é bem explorada [10]. Para o escoamento plano de Poiseuille, a instabilidade viscoelástica foi estudada recentemente, por Brandi, Mendonça e Souza [1]. Neste trabalho, os autores afirmam que, à medida que a quantidade da concentração de polímero no fluido diminui, o escoamento se torna mais estável, uma vez que o número crítico de Reynolds aumenta e a faixa de frequências instáveis diminui.

Quanto ao escoamento de jato viscoelástico e camada de mistura, Rallison e Hinch [5] consideraram o jato elástico submerso caracterizado pelo perfil parabólico para altos números de Reynolds e mostraram que o modo sinuoso está totalmente estabilizado por grande elasticidade, enquanto que o modo varicoso está parcialmente estabilizado. Mais recentemente, Zhang [10] investigou o escoamento do jato viscoelástico, empregando o modelo Oldroyd-B na equação constitutiva. Neste estudo, o resultado da análise de estabilidade modal em jatos poliméricos indicou que o efeito elástico não só afetou a instabilidade hidrodinâmica, aumentando o seu número de Reynolds crítico, mas também levou ao surgimento de um novo mecanismo de instabilidade, chamada de instabilidade elástica, para pequenos números de Reynolds.

\footnotetext{
${ }^{1}$ rlsterza@gmail.com.

2 analice.brandi@unesp.br.

${ }^{3}$ marciomtm@fab.mil.br.

${ }^{4}$ lefraso@icmc.usp.br.
} 
No entanto, considerando a Teoria de Estabilidade Linear para analisar as instabilidades do escoamento, é necessário conhecer o escoamento base do problema em questão. O escoamento base do jato laminar é dado por um perfil de velocidade canônico e os componentes do tensor extra-tensão são calculados considerando uma aproximação de escoamento paralelo, que foi validada comparando com os perfis do tensor extra-tensão obtidos por simulações computacionais de dinâmica dos fluidos. Diante disso, este trabalho objetiva verificar a hipótese de paralelismo pressuposta no escoamento base e, além disso, verificar as condições de estabilidade através de curvas neutras para diferentes parâmetros adimensionais.

\section{Formulação Matemática}

Esta seção apresenta as equações que modelam escoamentos isotérmicos e incompressíveis para fluidos não-Newtonianos. E, ainda, o modelo Oldroyd-B foi considerado para o tensor extra-tensão.

\subsection{Equações Governantes e Escoamento Base}

Considerando um escoamento incompressível e isotérmico de um fluido não-Newtoniano, as equações governantes adimensionais são dadas pelas equações de conservação de massa e do momento, isto é,

$$
\begin{aligned}
& \nabla \cdot \mathbf{u}=0 \\
& \frac{\partial \mathbf{u}}{\partial t}+\nabla \cdot(\mathbf{u u})=-\nabla p+\frac{\beta}{R e} \Delta \mathbf{u}+\nabla \cdot \mathbf{T},
\end{aligned}
$$

onde $\mathbf{u}$ denota o campo de velocidade, $t$ o tempo, $p$ a pressão, $\beta$ o coeficiente adimensional da viscosidade do solvente, $R$ e é o número de Reynolds e $\mathbf{T}$ é o tensor extra-tensão. Utiliza-se, neste trabalho, o modelo Oldroyd-B na equação constitutiva, dada na forma adimensional por

$$
\mathbf{T}+W i\left[\frac{\partial \mathbf{T}}{\partial t}+\nabla \cdot(\mathbf{u} \mathbf{T})-\mathbf{T}(\nabla \mathbf{u})^{T}-(\nabla \mathbf{u}) \mathbf{T}\right]=2 \frac{(1-\beta)}{R e} \mathbf{D},
$$

onde $W i$ é o número de Weissenberg e $\mathbf{D}=\frac{1}{2}\left(\nabla \mathbf{u}+(\nabla \mathbf{u})^{T}\right)$ é o tensor taxa de deformação.

Neste trabalho, os escoamentos de jatos viscoelásticos são estudados e as direções do fluxo do escoamento e a normal são dadas por $x$ e $y$, respectivamente. Considera-se, por hipótese, que o escoamento base é laminar e paralelo. A componente de velocidade do escoamento base é a mesma utilizada por Michalke (1971) [4] e os componentes do tensor extra-tensão não-Newtoniano do escoamento base são dados por

$$
U(y)=\frac{1}{2}\left[1+\tanh \frac{R}{4 \theta}\left(\frac{R}{y}-\frac{y}{R}\right)\right], \quad T_{b}^{y y}=0, \quad T_{b}^{x y}=\frac{(1-\beta)}{R e} \frac{d U}{d y} \quad \text { e } \quad T_{b}^{x x}=2 W i T_{b}^{x y} \frac{d U}{d y},
$$

onde $R$ denota a meia largura do jato e $\theta$ a espessura da camada limite. A fim de testar a aproximação do escoamento paralelo para os tensores extra-tensão, foram realizadas simulações numéricas de dinâmica de fluidos computacional através do software OpenFOAM. Os componentes do tensor extra-tensão resultantes da simulação utilizando o pacote CFD foram comparados aos perfis descritos na equação (4).

\subsection{Teoria de Estabilidade Linear}

A equação de Orr-Sommerfeld para um fluido viscoelástico do tipo Oldroyd-B foi apresentada por Souza et al (2016) [7]. O escoamento instantâneo é decomposto em duas partes, um escoamento 
base e um escoamento de perturbação. O escoamento base é considerado paralelo e invariante na direção $x$. As perturbações são escritas como modos normais

$$
\phi(x, y, t)=\bar{\phi}(y) e^{i(\alpha x-\omega t)},
$$

onde $\bar{\phi}$ representa a magnitude e a fase das perturbações, $i=\sqrt{-1}, \alpha=\alpha_{r}+i \alpha_{i}$ é o número de onda na direção $x$ e a taxa de crescimento espacial e $\omega$ é a frequência angular. Substituindo a solução do modo normal (5) nas equações de Navier-Stokes de perturbação e reescrevendo de forma simplificada, obtém-se a equação de Orr-Sommerfeld modificada para o fluido Oldroyd-B

$$
\begin{aligned}
& \frac{d^{4} v}{d y^{4}}-\left[i(\alpha U-\omega) \frac{R e}{\beta}+2 \alpha^{2}\right] \frac{d^{2} v}{d y^{2}}+\left[i \frac{\operatorname{Re} \alpha}{\beta} \frac{d^{2} U}{d y^{2}}+i \frac{\operatorname{Re} \alpha^{2}}{\beta}(\alpha U-\omega)+\alpha^{4}\right] v+ \\
& +\frac{R e}{\beta}\left[\alpha^{2} \frac{d T^{x x}}{d y}-i \alpha \frac{d^{2} T^{x y}}{d y^{2}}-i \alpha^{3} T^{x y}-\alpha^{2} \frac{d T^{y y}}{d y}\right]=0 .
\end{aligned}
$$

Esta equação é conhecida como equação de Orr-Sommerfeld para um fluido viscoelástico do tipo Oldroyd-B [7]. A solução da equação (6) corresponde a um problema de autovalor, sendo que a solução está diretamente ligada aos valores de $\alpha, \omega, R e, \beta$ e $W i$, e depende do perfil de velocidade do escoamento base em questão [1]. Os componentes do tensor extra-tensão não-Newtoniano, apresentados na equação (6), são dadas por

$$
\begin{aligned}
& T^{y y}(1-i(\omega-\alpha U) W i)=-W i\left(\frac{d T_{b}^{y y}}{d y} v-2 i \alpha v T_{b}^{x y}-2 \frac{d v}{d y} T_{b}^{y y}\right)+\frac{2(1-\beta)}{R e} \frac{d v}{d y}, \\
& T^{x y}(1-i(\omega-\alpha U) W i)=-W i\left(v \frac{d T_{b}^{x y}}{d y}-i \alpha v T_{b}^{x x}-\frac{d U}{d y} T^{y y}-\frac{i}{\alpha} \frac{d^{2} v}{d y^{2}} T_{b}^{y y}\right)+ \\
&+\frac{(1-\beta)}{R e}\left(i \alpha v+\frac{i}{\alpha} \frac{d^{2} v}{d y^{2}}\right), \\
& T^{x x}(1-i(\omega-\alpha U) W i)=-W i\left(v \frac{d T_{b}^{x x}}{d y}+2 T_{b}^{x x} \frac{d v}{d y}-2 T_{b}^{x y} \frac{i}{\alpha} \frac{d^{2} v}{d y^{2}}-2 T^{x y} \frac{d U}{d y}\right)-\frac{2(1-\beta)}{R e} \frac{d v}{d y} .
\end{aligned}
$$

Considerando as equações (7) - (9) juntamente com as condições de contorno apropriadas, obtêm-se os resultados da análise de estabilidade linear. A equação (6) também precisa ser avaliada nos limites para escoamentos de jato plano [2], as condições auxiliares são especificadas como segue

$$
U_{\infty}=U_{\text {coflow }}, \quad \text { para } y \rightarrow \pm \infty, \quad \text { e } \quad T_{b}^{x x}=T_{b}^{x y}=T_{b}^{y y}=0 .
$$

Além disso, todas as derivadas de $U$ e $T_{b}$ em relação a $y$ devem ser zero, pois $y \rightarrow \pm \infty$ e as perturbações decaem exponencialmente para longe da camada de cisalhamento do jato.

\section{Formulação Numérica}

Nessa seção são apresentadas as ferramentas utilizadas para verificar as hipóteses impostas no escoamento base, isto é, a utilização do software OpenFOAM e, além disso, a análise da solução numérica da equação de Orr-Sommerfeld.

\subsection{Software OpenFOAM}

O pacote CFD, do inglês Computational Fluid Dynamics, do OpenFOAM é ofertado gratuitamente e possui características particulares para solucionar numericamente as equações que modelam fenômenos da mecânica dos fluidos, em particular, as equações diferenciais parciais. O 
funcionamento do software é básico e pode ser visto no Guia do Usuário [9]. Neste problema, utilizou-se o solver viscoelasticFluidFoam sem alterações em seu código fonte.

Ressalta-se que, neste trabalho, utilizou-se o software OpenFOAM versão 7 e as principais alterações foram realizadas nos arquivos dos diretórios que descrevem o domínio (foi necessário fazer um adensamento de malha na região $y$ ) e os componentes das condições de entrada e contorno (utiliza-se a velocidade descrita na equação (4) e condições para a pressão e para os tensores).

\subsection{Solução Numérica da Equação de Orr-Sommerfeld}

A equação de Orr-Sommerfeld, obtida através da Teoria de Estabilidade Linear, representa um problema de autovalor e, neste trabalho, foi resolvida através do método Shooting. O código implementado, resumidamente, utiliza Runge-Kutta de quarta ordem para integrar a equação de Orr-Sommerfeld, onde a frequência $\omega$, os parâmetros adimensionais $R e, W i$ e $\beta$ e uma estimativa inicial para $\alpha$ são dadas. A integração ocorre nos limites $y= \pm \infty$ para o centro $y=0$. As autofunções resultantes são comparadas e utiliza-se o método da Secante para corrigir a estimativa para $\alpha$ até que a comparação entre as duas autofunções atinja um valor de tolerância pequeno. Durante a integração, um procedimento de ortonormalização de Gram-Schmidt é usado para garantir que os autovetores viscosos e invíscidos permaneçam ortogonais [3].

$\mathrm{Na}$ região onde o escoamento laminar se torna instável, dois tipos de flutuação da velocidade sinusoidal foram encontrados: um é simétrico e o outro é anti-simétrico em relação à linha central do jato [6]. Em outras palavras, o escoamento de jato está sujeito as instabilidades de KelvinHelmholtz, a partir de dois modos instáveis independentes, conhecidos como varicoso (simétrico) e sinuoso (anti-simétrico).

\section{Resultados Numéricos}

Nesta seção são apresentados os resultados numéricos do estudo do comportamento do escoamento de jato laminar em termos de como evolui a jusante, utilizando o software OpenFOAM para simulá-lo numericamente.

\subsection{Estudo do Escoamento Laminar}

Nesta seção apresenta-se uma investigação do comportamento do escoamento de jato laminar através da comparação dos tensores não-Newtonianos, que foram considerados para a análise de estabilidade, e os obtidos pelo OpenFOAM com o objetivo de verificar, por exemplo, a hipótese de escoamento paralelo. No trabalho de Sterza (2020) [8] há uma verificação da metodologia utilizando o escoamento de Poiseuille, onde a solução analítica é conhecida. A Figura 1 ilustra o domínio do escoamento do jato, sendo $L$ o comprimento do escoamento e $H$ a altura.

O objetivo dessa seção é realizar a comparação dos tensores $T_{\text {foam }}$ e $T\left(U_{\text {foam }}\right)$, tais que $T_{\text {foam }}$ representam os tensores simulados do OpenFOAM enquanto $T\left(U_{\text {foam }}\right)$ são os tensores calculados através da equação (4), onde a velocidade utilizada foi obtida da simulação realizada no software OpenFOAM. Vale destacar que, as derivadas da velocidade foram calculadas por aproximações de quarta ordem e, além disso, os resultados do OpenFOAM foram obtidos na posição $x=\frac{2}{3} L$.

Por consequência, alguns parâmetros fixos são considerados: $L=90$ e $H=30$, além disso, a velocidade de entrada (inlet) é a apresentada na equação (4) para $R=1, \theta=0.1$ e $U_{\text {coflow }}=0.1$, permitindo que o software realize a simulação até atingir o estado estacionário. Os resultados foram simulados para diferentes parâmetros adimensionais, mostrando boa concordância entre os resultados obtidos utilizando o pacote CFD e os valores de aproximação de escoamento paralelo. 


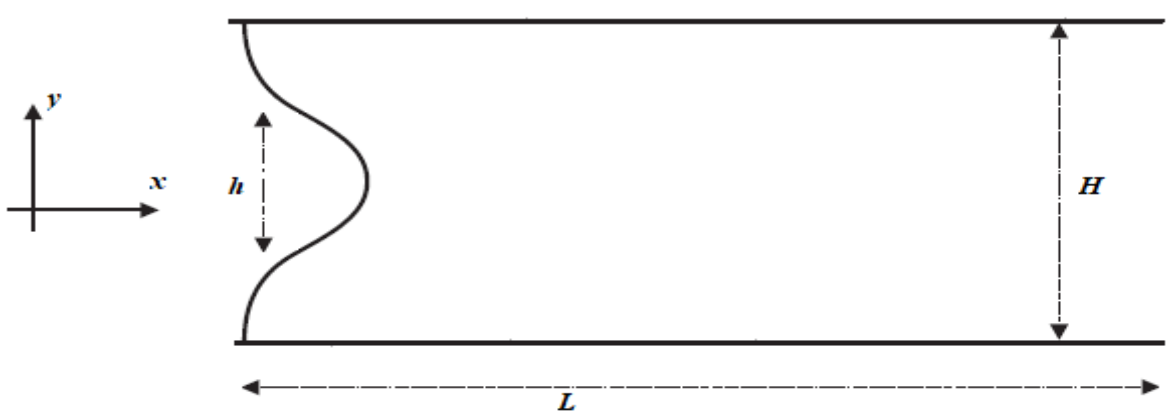

Figura 1: Domínio computacional para o escoamento do jato.

Os resultados da Figura 2 são para $R e=250, \beta=0,5$ e $W i=6$ e $W i=10$, sendo que a Figura 2(a) e a Figura 2(b) referem-se aos tensores não-Newtoniano $T^{x x}$ e $T^{x y}$, respectivamente.

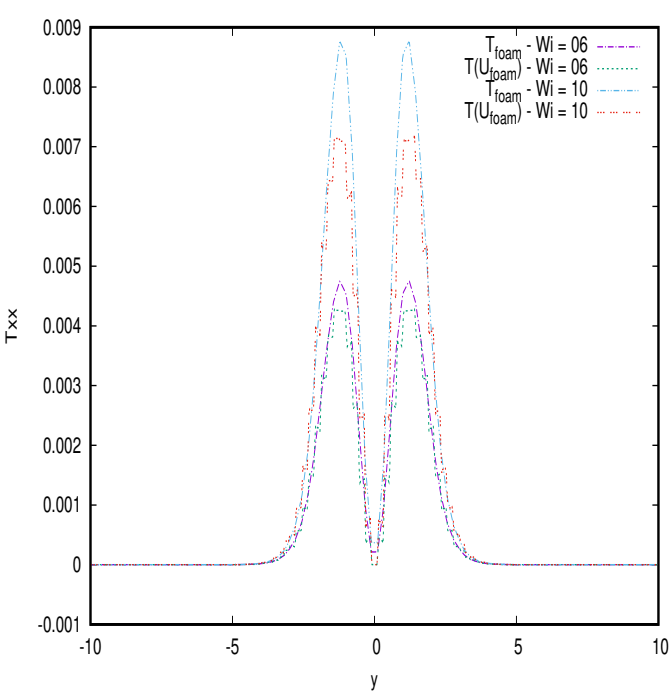

(a)

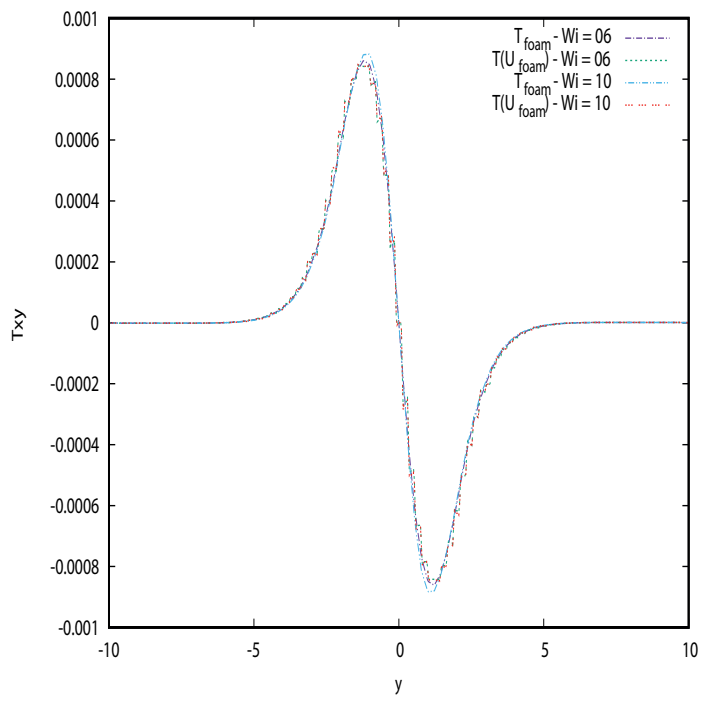

(b)

Figura 2: Tensores não-Newtonianos para $R e=250, \beta=0.5, W i=6$ e $W i=10$ : (a) $T^{x x}$ e (b) $T^{x y}$.

Os resultados mostram uma boa concordância entre os tensores calculados, $T^{x x}$ e $T^{x y}$, e os tensores correspondentes calculados com a suposição de escoamento paralelo. O tensor $T^{x y}$ não varia com o aumento do número de Weissenberg, conforme esperado pela equação (4). As pequenas diferenças na amplitude podem ser atribuídas à resolução do domínio computacional dos resultados obtidos utilizando o pacote CFD. Além disso, os resultados de $T^{y y}$ ficaram próximos de zero, por isso, foram omitidos.

\subsection{Análise de Estabilidade: Curvas Neutras}

Os resultados da análise espacial utilizando o código de Teoria de Estabilidade Linear são apresentados nesta subseção através das curvas neutras de estabilidade. Em Sterza (2020) [8] 
realizou-se uma verificação do código numérico através de comparações de curvas neutras encontradas na literatura. Nesta análise considera-se $\omega$ real e $\alpha$ complexo, isto é, uma análise espacial. Com o objetivo de avaliar as curvas neutras de estabilidade, diferentes simulações numéricas foram realizadas variando-se os parâmetros adimensionais para o escoamento do fluido Oldroyd-B e comparando com o escoamento de fluido Newtoniano. Neste trabalho, os parâmetros estudados são o número de Reynolds $(R e)$, o número de Weissenberg $(W i)$ e a constante $\beta$.

As curvas neutras separam as regiões estáveis de taxa de amplificação negativa $-\alpha_{i}<0$ (acima da linha neutra), das regiões instáveis de taxa de crescimento positiva $-\alpha_{i}>0$ (abaixo da linha neutra). Os componentes do escoamento base para velocidade e os tensores não-Newtonianos utilizados são dados pela equação (4) para $R=1$ e $\theta=0.1$ e, ainda, $y \in(-4,4)$. A Figura 3 apresenta as curvas neutras de estabilidade para o modo sinuoso e varicoso utilizando $\beta=0.5 \mathrm{e}$ $\beta=0.7$, variando o número de Weissenberg e, também, apresenta a curva neutra para o fluido Newtoniano.

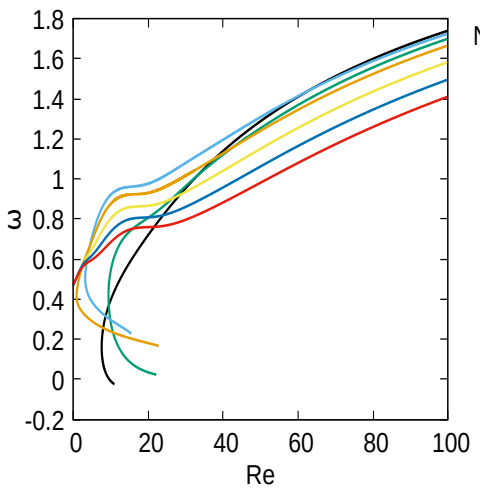

(a) Modo sinuoso e $\beta=0.5$.

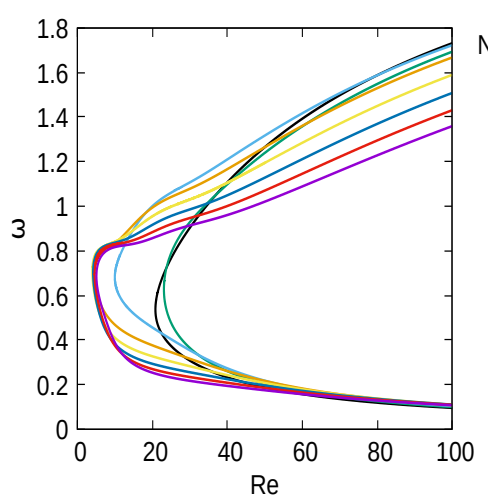

(c) Modo varicoso e $\beta=0.5$.
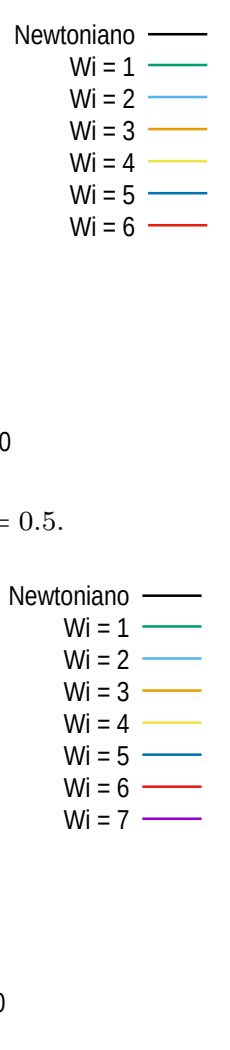

Figura 3: Curvas neutras de estabilidade para os modos instáveis de Kelvin-Helmholtz.

Observa-se um comportamento semelhante entre os dois modos de instabilidade, sendo que os efeitos elásticos (aumento de $W i$ ) e a diminuição da contribuição do solvente Newtoniano no fluido (diminuição de $\beta$ ) contribuíram para o aumento das regiões instáveis. Pode-se questionar o motivo do ramo inferior da curva neutra, no caso sinuoso, não ser completa. Verificou-se a aproximação do modo sinuoso com o espectro contínuo e, com isso, o código implementado não consegue distinguir qual é o modo sinuoso e qual é o espectro contínuo, impedindo a completa construção da curva neutra. Além disso, Zhang (2012) [10] descreve o surgimento de instabilidades elásticas nesses 
problemas.

\section{Conclusões}

Neste trabalho, apresenta-se as equações adimensionais que modelam escoamentos de jatos incompressíveis, isotérmicos e bidimensional para fluidos não-Newtonianos, em particular, viscoelástico, empregando o modelo Oldroyd-B como equação constitutiva. Primeiramente, utiliza-se o software OpenFOAM para simular numericamente o escoamento de jato laminar, com o objetivo de confirmar a hipótese de escoamento paralelo imposta para a escolha do escoamento base e na utilização da aproximação canônica para o componente de velocidade. De certo modo, observou-se que as pressuposições foram adequadas ao problema proposto. Em seguida, investiga-se os dois modos de instabilidade de Kelvin-Helmholtz, sinuoso e varicoso, sendo que esses apresentam alguns problemas de convergência, uma vez que o espectro contínuo está muito próximo do modo instável e, também, pode estar surgindo instabilidades elásticas, que não foram analisadas, conforme proposto na literatura.

\section{Agradecimentos}

Agradecemos à CAPES pelo auxílio financeiro no desenvolvimento deste trabalho.

\section{Referências}

[1] Brandi, A. C., Mendonça, M. T. and Souza, L. F. DNS and LST stability analysis of OldroydB fluid in a flow between two parallel plates. Journal of Non-Newtonian Fluid Mechanics, 267:14-27, 2019. DOI: 10.1016/j.jnnfm.2019.03.003.

[2] Kundu, P. and Cohen, I. Fluid mechanics, 4th. edition. Academic Press, Kidlington, 2010.

[3] Mendonça, M. T. e Medeiros, M. A. F. Estabilidade de Escoamentos Laminares: Teoria Linear, volume 2, ABCM, Uberlândia, 2000.

[4] Michalke, A. Instabilität eines kompressiblen runden Freistrahls unter Berücksich-tigung des Einflusses der Strahlgrenzschichtdicke. Progress in Aerospace Sciences, 19:319-328, 1971. English Translation: 1977, NASA Technical Memorandum 75190.

[5] Rallison, J. M. and Hinch, E. J. Instability of a high-speed submerged elastic jet. Journal Fluid Mech, 288:311-324, 1995. DOI: 10.1017/S0022112095001157.

[6] Sato, H. The stability and transition of a two-dimensional jet. Journal of Fluid Mechanics, 7:53-80, 1960. DOI: 10.1017/S0022112060000049.

[7] Souza, L. F., Brandi, A. C. e Mendonça, M. T. Estabilidade de Escoamentos de Fluidos não-Newtonianos, Turbulência, ABCM, volume 10, capítulo 2, p. 101-160, 2016.

[8] Sterza, R. L. Investigação da estabilidade de escoamentos de jatos viscoelásticos, Dissertação de Mestrado, Universidade Estadual Paulista, 2020.

[9] The OpenFOAM Foundation. OpenFOAM v'7 User Guide, OpenCFD Ltd, 2019.

[10] Zhang, M. Linear stability analysis of viscoelastic flows, Master's Dissertation, Royal Institute of Technology, 2012. 